\title{
Efficient Real-Time Industrial Ethernet Links
}

\author{
P. Rahul Reddy \\ ${ }^{1}$ Associate Professor, Department of ECE, Swami Ramananda Tirtha Institute of Science \&Technology, Nalgonda, India
}

\begin{abstract}
Real-time Industrial Ethernet (RE), especially IEC 61158, plays increasing role in industrial communications these days, so our motto is to concentrate on Energy efficiency of interest for industrial communications. The alteration introduces a new operational mode, well-defined as Low Power Idle (LPI) that permits to noticeably reduce the power consumption of inactive Ethernet links. We flinch with an explanation of the EEE basics and, afterward, emphasis on the overview of EEE in the industrial communication scenario. Then, we explicitly address the carrying out of effective EEE strategies for some widespread RTE networks.
\end{abstract}

Keywords: Energy efficient Ethernet (EEE), industrial automation, real-time Ethernet (RTE) networks

\section{Introduction}

Energy consumption is nowadays a global source of concern for both economic and environmental reasons. Networking equipment alone consumes $1.8 \%$ of the world's electricity, and that number is currently increasing at a $10 \%$ rate annually [1]. If we just focus on data centers, between 15 and $20 \%$ of electricity is used for networking [2]. These reasons are spurring the development of more power efficient networking equipment. A direct result of these efforts is the IEEE 802.3az standard [3] which provides a new idle mode for Ethernet physical interfaces. This new mode only needs a small fraction of the power used in normal operation, but no traffic can be transmitted nor received while the interface stays in the idle mode. Since there is an implicit trade-off between energy consumption and frame delay, these new Energy Efficient Ethernet (EEE) interfaces need a governor that decides when to enter and exit this idle mode. In fact, several alternatives have already been proposed in the literature [4], [5] and have been later validated by both empirical and analytic.

Industrial versions of Ethernet networks [6] are playing an ever more important role, thanks to their intrinsic valuable features that allow obtaining isochronous real-time communications [7], [8]. These networks usually referred to as Real-Time. Ethernet (RTE), are envisaged to have a considerable growth in the next years [9]. Moreover, their transmission rate, which is currently $100 \mathrm{Mb} / \mathrm{s}$ for almost all the available protocols, will be increased up to 1 Gbps or even $10 \mathrm{~Gb} / \mathrm{s}$ in the future. Thus, very likely EEE concepts will be of strong interest for RTE networks as well. In this field of application, however, the time overheads introduced by the LPI mode may reveal critical, since industrial communication applications often require a tight timing. On the other hand, it has to be considered that industrial traffic is to a considerable extent predictable, as it is often generated by cyclic operations [10]. Such knowledge reveals of great help to design timely and effective EEE strategies.

In this paper, we hence focus on the adoption of EEE by RTE networks. After an assessment of the LPI mode that includes a thorough timing analysis, we will concentrate on the design of effective EEE strategies for some of the most popular RTE networks.

\section{Literature Review}

In [11], the authors analyze the application of appropriate EEE strategies to Ethernet POWERLINK, a popular RTE network. The results, obtained from theoretical analyses, are encouraging, since the achievable power saving demonstrated to be considerable.

Focusing on the same RTE network, paper [12] provides a simulation study that investigates further EEE strategies aimed at harmonizing power savings and timing requirements.

Another contribution, although not officially published, is given in [13].In this case, the authors provide a feasibility study about the possibility of adapting the EEE philosophy to RTE networks, proposing to incorporate them in the IEEE 802.3az amendment. Unfortunately, the proposal has not been further discussed, even if it represents a valuable attempt to address the topic from a standardization point-ofview.

Indeed, some of them are recalled in [14], where the authors focus on sleeping algorithms as a way to provide high power saving, while ensuring the unavoidable delays that influence frame transmission are kept very low.

Similarly, in[15], a technique called packet coalescing is proposed. Here, the authors concentrate on the transmission of large TCP frames and show the benefits, in terms of energy efficiency, of grouping acknowledgment frames before their actual transmission, instead of sending them one by one immediately.

Another interesting contribution is given in [16]. In this case, the authors provide an analytical model to evaluate the time spent by a link in the various states defined by EEE. Such a time is the key metric necessary to calculate power consumption as well as to design traffic shaping strategies to increase power saving. The model, developed under the assumption that frames arrive as batches with Poisson distribution, has been validated using real traffic traces.

As a further issue, power savings provided by EEE could reveal of interest for some new Ethernet amendment proposals, currently under discussion within the IEEE 802.3 working group [17]. 


\section{International Journal of Science and Research (IJSR) \\ ISSN (Online): 2319-7064 \\ Index Copernicus Value (2013): 6.14 | Impact Factor (2014): 5.611}

Finally, it is worth stressing that energy efficient communication systems are expected to play an important role in "green manufacturing" [18], a driving concept for the design and implementation of innovative manufacturing systems, where priority is given to aspects like both reduction of the environmental impact and use of natural resources.

\section{System Methodology}

EEE is based on the overview of the LPI mode, a new article of Ethernet devices that permits Ethernet links to cross the threshold anew functional state, specifically, quiet state in which power consumption is noticeably reduced and communication is not permissible (only minimal signaling on the medium is maintained). In this course, the medium access control (MAC) protocol has-been altered with the introduction of the "LPI client" stack, as shown in Fig. 1.

Data Link Layer
Logical Link Control
$\begin{gathered}\text { Low Power Idle } \\ \text { (LPI) Client }\end{gathered}$ Medium Access
Control (MAC)

Figure 1: Protocol architecture of a station implementing EEE

So therefore an innovative MAC architecture makes the LP_IDLE protocol service accessible to the upper layers that can feat it to implement EEE strategies. Certainly, the transition to/from quiet state takes place via both request and indication primitives of such a protocol service, as pronounced in Fig. 2

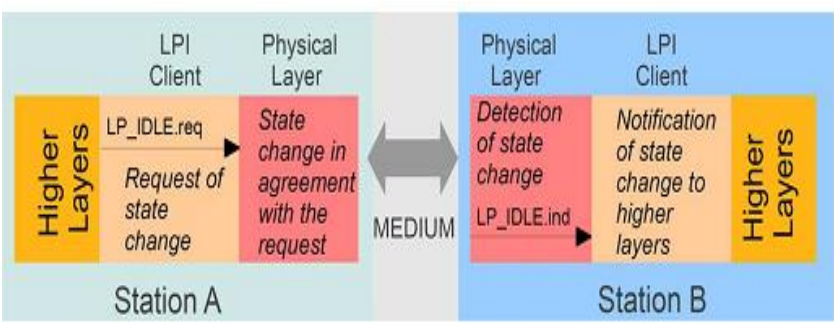

Figure 2: LPI client protocol primitives.

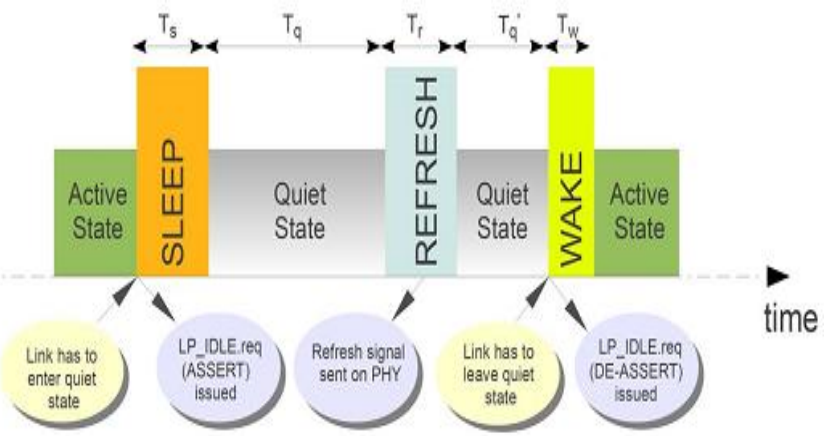

Figure 3: Example of LPI operation

When one of the partners of an Ethernet link (e.g., Station A in Fig. 2) agrees to strengthen the link in quiet state, it invokes the LP_IDLE. Request primitive with the (unique) parameter LPI_REQUEST set to ASSERT.

At that moment, the PHY starts transitioning to quiet state. The PHY of the destination partner (Station B) notices the new state of the link listening to the transmission medium and, accordingly, issues the LP_IDLE. Indication primitive to its LPI client which, in turn, notifies the higher layers about the link state change. The same process is followed to wake up the link but, in this case, the LPI_REQUEST parameter has to be set to DE-ASSERT. It is worth emphasizing that the procedure of altering the link state is conveyed completely through electrical signaling on the link itself, and no packet transmission is involved.

When oneof the partners issues the LPI REQUEST
primitive, its specificsignaling indicates the request
to the other link partner that hasto implicitly
agree.IEEE 802.3 az provides also some important
information concerning the timing of the LPI mode
operation. In particular, thetime necessary to an
active link to move to quiet state is definedas sleep
time, $\mathrm{T}_{5}$. The time spent in quiet state is application
dependent, but it is required a refresh signal is
issued with fixedperiod $\mathrm{T}_{a}$. The duration of the
refresh procedure is $\mathrm{T}_{\mathrm{s}}$. Finally,stepping back from
quiet to active state requires a time definedas wake
time, An example of LPI operation behavior is
shown in Fig. 3 . Ascan be seen, the example refers
to a link that initially switchesfrom active to quiet
state upon the request of one of its partners. The link
remains in such a state for a time $T_{a}$. Then, the
refresh procedure takes place. Subsequently, after,
a new statechange is requested to the active state,
which is reached after $\mathrm{T}_{w}$

Application of EEE to RTE networks: The distinctive real-time industrial traffic may be subdivided in two classes, explicitly, cyclic and acyclic.

Cyclic: It is produced by operations like set-point transmission, periodic sampling of sensor data and, in general, repetitive actions.

Acyclic: In this traffic originates from unpredictable events such as, for instance, those related to process alarms.

\section{Implementation of System}

We studied some sort of Significance of Real-Time Industrial Ethernet that is mentioned below:

PROFINET IO is a extensive RTE network comprehended by the IEC 61784-2 International Standard, existing in two forms, namely:

1) PROFINET IO IRT (Isochronous Real-Time), referred to as communication profile $\mathrm{CP}$ 3-6 by the standard, and

2) PROFINET IO RT (Real-Time) that covers the communication profiles CP 3-4 and CP 3-5.

Two main types of devices are employed by both versions of PROFINET IO. They are, namely: 


\section{International Journal of Science and Research (IJSR) \\ ISSN (Online): 2319-7064 \\ Index Copernicus Value (2013): 6.14 | Impact Factor (2014): 5.611}

1) IO controllers (IOCs, typically intelligent devices like PLCs or PCs implementing automation tasks) and

2) IO devices (IODs, field devices like, for example, sensors and/oractuators).

We found that in Industrial (RE!) Ethernet $<2 \%$ of total Ethernet market. But: \# of installed units rapidly increasing Ethernet: 10\% p.a and similarly Industrial Ethernet :> 30\% p.a.!

\begin{tabular}{|c|c|c|}
\hline $\begin{array}{c}\text { Network } \\
\text { structure }\end{array}$ & $\begin{array}{c}\text { Office Networks } \\
\text { Core, distribution, } \\
\text { access (typically } \\
\text { star topology) }\end{array}$ & $\begin{array}{c}\text { Industrial Networks } \\
\text { machine level (typically } \\
\text { line or ring) }\end{array}$ \\
\hline $\begin{array}{c}\text { Real time } \\
\text { communication }\end{array}$ & $\begin{array}{c}\text { Minimum response } \\
\text { time up } \\
\text { to } 150 \mathrm{~ms}\end{array}$ & $\begin{array}{c}\text { Response-time } \\
\text { requirements 10's of } \mu \mathrm{s}-\sim \\
100 \text { ms (rather on the } \\
\text { shorter side) }\end{array}$ \\
\hline $\begin{array}{c}\text { Environmental } \\
\text { conditions }\end{array}$ & $\begin{array}{c}\text { Office" conditions } \\
\text { for network } \\
\text { elements }\end{array}$ & $\begin{array}{c}\text { Harsh industry atmosphere } \\
\text { (moisture, dust, shock, ...) }\end{array}$ \\
\hline $\begin{array}{c}\text { Availability } \\
\text { and redundancy }\end{array}$ & $\begin{array}{c}\text { OSPF failover times } \\
1-10 \text { s; } \sim 3 s f o r \text { RSTP }\end{array}$ & $\begin{array}{c}\text { Failover times < 200 ms } \\
\text { with } \\
\text { MRP (IEC 62349) }\end{array}$ \\
\hline
\end{tabular}

\section{Discussions}

The following Example according to IEC 61158 gives some idea about energy savings in industrial communications: $\mathrm{T}_{\mathrm{q}}$

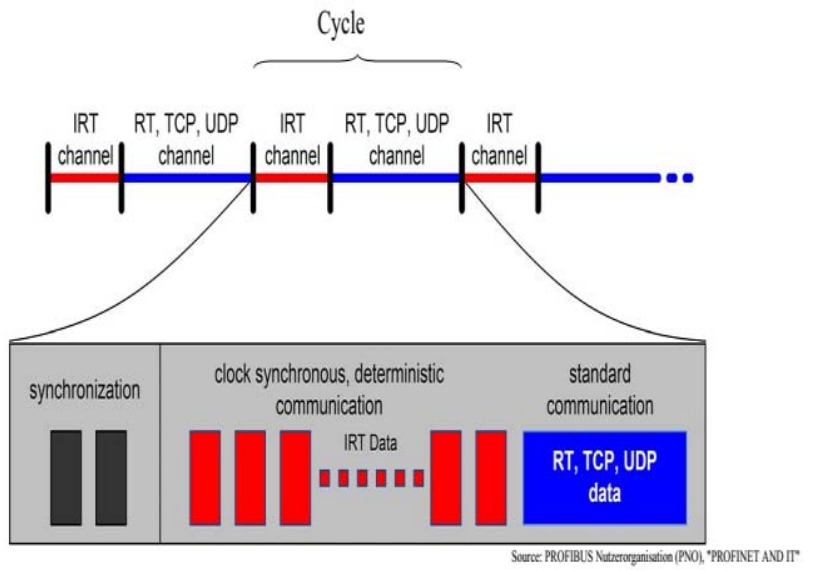

Figure 4: Time-slotted data transfer

We are saving energy in industrial communications due to following reasons:

a) Fixed or lessening thermal budget and size of components

- Passive cooling only, no air vents and Denser packing made promising through lower energy consumption.

- In contrast/conflict with data-rate increase (FE -> GE), Cost saving (energy expenditure) as well as Industrial networks continually on but not always fully used (lunch break, overnight, weekends, holidays...)

b) Global energy conservation (Green ICT)

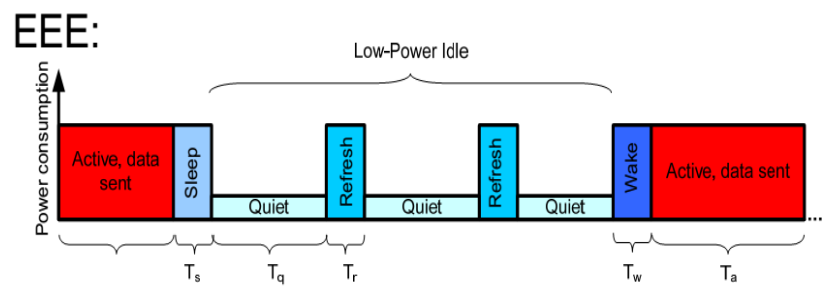

What we have:

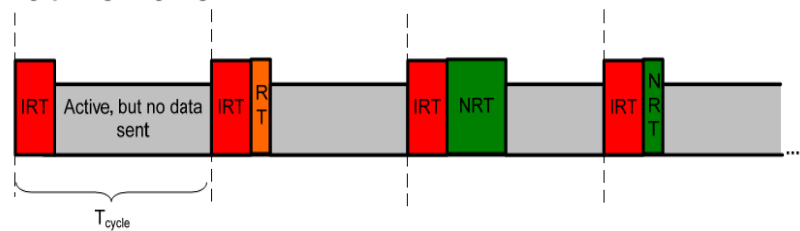

Figure 5:

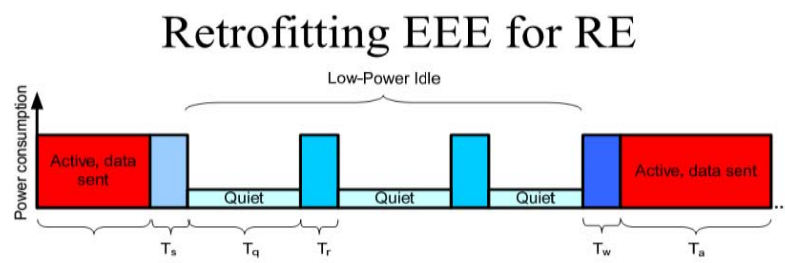

One approach: shorter low-power idle within each cycle!

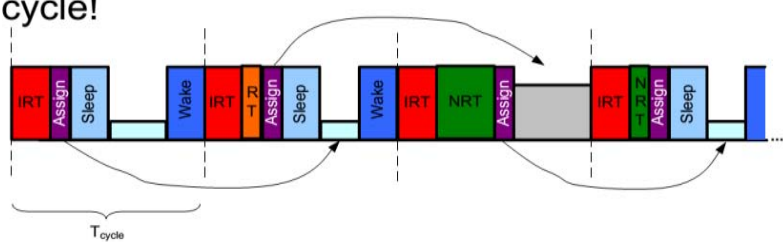

Figure 6:

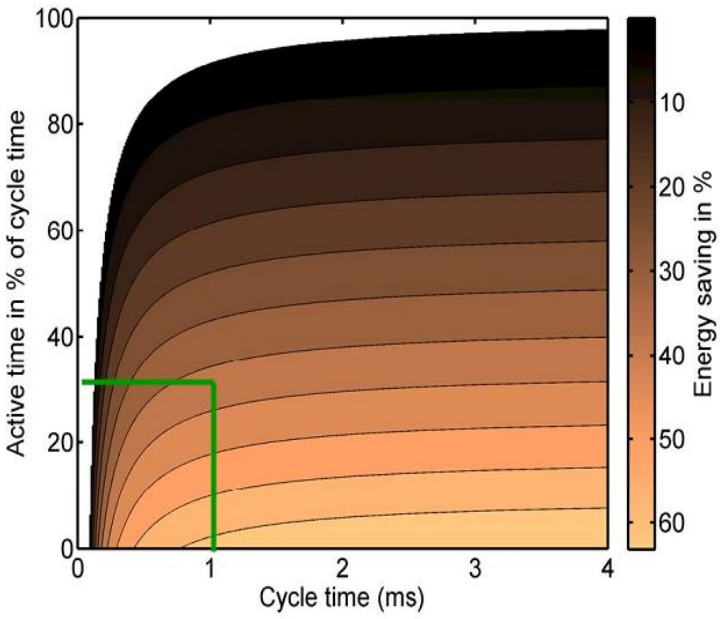

Figure 7

Expected energy savings from retrofitted EEE in 100BaseTX Timers taken from draft 1.1 • Energy consumption taken from Chou et al., Jan2008

\section{Conclusion}

In this paper, we provided a valuation of the problems troubled with the application of EEE to industrial networks. The analysis started with a explanation of the IEEE 802.3az adjustments. Then, we really addressed RTE networks and examined how they could adopt effective EEE strategies. The investigation proved that significant power savings can be accomplished; actually preserving the high-performance 


\section{International Journal of Science and Research (IJSR) \\ ISSN (Online): 2319-7064 \\ Index Copernicus Value (2013): 6.14 | Impact Factor (2014): 5.611}

level these networks are demanded to provide. Subsequently, we rely on the summary of EEE in the industrial communication scenario is an encouraging research topic.

\section{References}

[1] S. Lambert, W. V. Heddeghem, W. Vereecken, B. Lannoo, D. Colle, and M. Pickavet, "Worldwide electricity consumption of communication networks," Optics Express, vol. 20, no. 26, pp. 513-524, Dec. 2012.

[2] B. Heller, S. Seetharaman, P. Mahadevan, Y. Yiakoumis, P. Sharma,S. Banerjee, and N. McKeown, "Elastictree: Saving energy in data center networks," in USENIX Symposium on Networked Systems Design and Implementation, NSDI 2010, San Jose, CA, USA, Apr. 2010, pp. 249-264.

[3] “IEEE 802.3az," Oct. 2010. [Online]. Available: http://dx.doi.org/10.1109/IEEESTD.2010.5621025

[4] M. Gupta and S. Singh, "Using low-power modes for energy conservation in Ethernet LANs," in Proceedings of the IEEE INFOCOM, Anchorage, AK; USA, 2007, pp. 2451-2455.

[5] M. Rodriguez Perez, S. Herreriı Alonso, M. Fernandez Veiga, and 'C. Lopez Garcia, "Improved opportunistic sleeping algorithms for LAN switches," in Proceedings of the IEEE Globecom, Honolulu, HI, USA, Dec. 2009.

[6] "Industrial Communication Systems," in The Industrial Information Technology Handbook, R. Zurawski, Ed. Boca Raton, FL, USA: CRC Press, 2005, pp. 37.147.16.

[7] J. D. Decotignie, "Ethernet-based real-time and industrial communications," Proc. IEEE, vol. 93, no. 6, pp. 1102-1117, Jun. 2005.

[8] M. Felser, "Real-time Ethernet-industry prospective," Proc. IEEE, vol. 93, no. 6, pp. 1118-1129, Jun. 2005.

[9] Industrial Ethernet Book, no. 69/72, Apr.-Oct. 2012. [Online]. Available: http://www.iebmedia.com.

[10]T. Sauter, "The three generations of field-level networks-evolution and compatibility issues," IEEE Trans. Ind. Electron., vol. 57, no. 11, pp. 3585-3595, Nov. 2010.

[11]J. A. Maestro and P. Reviriego, "Energy efficiency in industrial Ethernet: The case of POWERLINK," IEEE Trans. Ind. Electron., vol.57, no. 8, pp. 2896-2903, Aug. 2010.

[12]F. Tramarin and S. Vitturi, "Energy efficient Ethernet for the industrial communication scenario," in Proc. IEEE 18th Conf. Emerging Technol. Factory Autom. (ETFA), 2013, pp. 1-8.

[13] J. W. Walewski, V. Kulkarni, and G. Steindl, in EEE for Real-Time Industrial Ethernet, presented at the IEEE802.3 Meeting, Vancouver, Canada, 2009. [Online]. Available: http://www.ieee802.org/3/az/public/mar09/walewski_01 _0309.pdf.

[14] $\bar{S}$. Herreria-Alonso, M. Rodriguez-Perez, M. FernandezVeiga, and C.Lopez-Garcia, "Optimal configuration of energy-efficient Ethernet,” Comput. Networks, vol. 56, no. 10, pp. 2456-2467, 2012.

[15] K. Christensen, P. Reviriego, B. Nordman, M. Bennett, M. Mostowfi, and J. Maestro, "IEEE 802.3az: the road to energy efficient Ethernet, "IEEE Commun. Mag., vol. 48, no. 11, pp. 50-56, Nov. 2010.

[16] M. A. Marsan, A. Anta, V. Mancuso, B. Rengarajan, P. Vasallo, and G.Rizzo, "A simple analytical model for energy efficient Ethernet," IEEE Commun. Lett., vol. 15, no. 7, pp. 773-775, Jul. 2011.

[17] L. Winkel, "Distinguished minimum latency traffic in a converged traffic environment, DMLT," Jul. 2013 [Online].

Available: http://www.ieee802.org/3/DMLT/public/Jul13/Winkel_ 00_0713_DMLT_SG_Tutorial_v04.pdf.

\section{Author Profile}

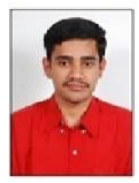

P. Rahul Reddy attained his B.Tech in Electronics \& Communication Engineering and M.Tech in the stream of Embedded Systems from JNTU, Hyderabad. He is having teaching experience of more than 5 years in various Under Graduate and Post Graduate course. He has guided lots of students in various Under Graduate and Post Graduate Research Projects. At present, he is working as Associate Professor, Department of ECE in Swami Ramananda Tirtha Institute of Science \& Technology, Nalgonda, India. 